\title{
Kulturwissenschaft
}

\author{
Matthias Weber
}

Bundesinstitut für Kultur und Geschichte der Deutschen im östlichen Europa, Oldenburg

DOI: $10.19195 / 0435-5865.141 .26$

\section{Kultur und Geschichte der Deutschen im östlichen Europa im deutsch-polnischen Diskurs. Über Asymmetrien des historischen Erinnerns}

\section{Vorbemerkung: Deutsche im östlichen Europa}

Die Perspektiven von Deutschen und Polen auf die Vergangenheit sind unterschiedlich. ${ }^{1}$ Die im Zweiten Weltkrieg zugefügten Wunden sind auch sieben Jahrzehnte nach Kriegsende nicht verheilt. Das haben die Diskussionen über den Film „Unsere Mütter, unsere Väter“ gezeigt: Während das Zweite Deutsche Fernsehen (ZDF) den im März 2013 ausgestrahlten Dreiteiler ganz unpolitisch als eine Chance betrachtete, die Generation der Teilnehmer am Zweiten Weltkrieg mit ihren Kindern, Enkeln und Urenkeln in einen Dialog zu bringen, wurde in den polnischen Medien und sogar auf Seiten der Regierung Kritik geäußert: Die polnischen Partisanen wie die Zivilbevölkerung würden einseitig als primitiv und als Judenhasser gezeigt; der Film würde den guten Namen der Heimatarmee verletzen. Nach Ausstrahlung der Serie in Polen (Juni 2013; 3,7 Millionen Zuschauer) erreichte die Entrüstung den Höhepunkt: Vorwürfe der Relativierung der Kriegsschuld Deutschlands und der Schuld am Holocaust waren zurück. ${ }^{2}$

${ }^{1}$ Überarbeitete und aktualisierte Fassung des Kapitels Germans in Eastern Europe as a Polish-German Lieu de mémoire. On the Asymmetry of Memories. In: Pakier, Małgorzata, Wawrzyniak, Joanna (Hrsg.): European Memories - The Eastern Perspective (Contemporary European History). New York 2015. S. 264-282.

${ }^{2}$ Vgl. die Informationen in der ZDF-Mediathek zum Film http://umuv.zdf.de/Unsere-M\%C3\%BCtter-unsere-V\%C3\%A4ter/Unsere-M\%C3\%BCtter-unsere-V\%C3\%A4ter-26223848.html [Zugriff am 01.10.2015]. Dokumentation der FRANKFURTER ALLGEMEINEN ZEITUNG http://www.faz. net/aktuell/feuilleton/medien/unsere-muetter-unsere-vaeter/ [Zugriff am 01.10.2015], und GAZETA WYBORCZA "Nasze matki, nasi ojcowie" - skandal czy serial poruszajacy, vgl. http://wyborcza. pl/1,75968,14141962,_Nasze_matki_nasi_ojcowie__skandal_czy_serial_poruszajacy.html\#ixzz2c7sHd300 [Zugriff am 01.10.2015]; Steve Robson: Fury in Poland over German war drama which 
Die Filmemacher hatten zumindest die notwendige Sensibilität vermissen lassen. Vielleicht fehlten auch einfach nur die Kenntnisse über die Verbrechen, die im Zweiten Weltkrieg von Deutschen in und an Polen verübt worden waren und über die heutige Präsenz der Vergangenheit in der polnischen Gesellschaft. Bei der Wiederholung des Films 2015 im Kultursender 3SAT wurde nachfolgend eine Dokumentation ins Programm aufgenommen, die auch das polnische Leid thematisierte: „Kampf ums Überleben - Polen unter Deutscher Besatzung“. Die Neuausstrahlung geschah im Rahmen des TV-Themenschwerpunkts anlässlich des 2015 stattfindenden siebzigsten Jahrestags des Kriegsendes. Im August 2015 beendete das ZDF diesen Schwerpunkt mit dem polnischen Spielfilm „Warschau '44“ (Original „Miasto '44“) über den Warschauer Aufstand von 1944, der ein Jahr zuvor im Warschauer Stadion erstaufgeführt worden war. „Die Deutschen sollen sich unbedingt diesen Film ansehen“, schrieb danach der Journalist Tomasz Lis, denn sie würden die Polen so lange nicht verstehen können, bis sie erführen, was damals geschah und was der Warschauer Aufstand bis heute für Polen bedeutet. ${ }^{3} \mathrm{Um}$ sicher zu gehen, dass die Deutschen den Film richtig einordnen, zeigte das ZDF im Anschluss wieder eine Dokumentation, um zu erklären, warum der Aufstand gegen die deutsche Besatzung bis heute für Polen ein nationales Trauma ist. Im Abendprogramm des ZDF erreichte „Warschau '44“" aber nur schwache Einschaltquoten (1,06 Millionen Zuschauer), ,und bestätigte einmal mehr die Asymmetrie der gegenseitigen Wahrnehmung von Polen und Deutschen“. ${ }^{4}$

Die Deutschen blicken auf eine sehr lange Geschichte im östlichen Europa und besonders in Polen zurück. Den während des Zweiten Weltkriegs an Polen und in Polen begangenen Verbrechen - Okkupation, Unterdrückung und Massenmord waren Jahrhunderte friedlicher Koexistenz vorangegangen, die von wechselseitigem kulturellem Austausch, Wissens- und Kulturtransfer und immer wieder auch von Konkurrenzen und Konflikten geprägt waren. Als Ergebnis haben Deutsche und Polen mit Angehörigen anderer Ethnien gemeinsam ein reiches kulturelles Erbe geschaffen: in der Kunst, in der Literatur oder in der Architektur. Umsiedlungen sowie Flucht und Vertreibung der Deutschen vor, während und nach dem Zweiten Weltkrieg beendeten die Anwesenheit der Deutschen im östlichen Europa weitgehend.

'tries to spread blame for Holocaust', vgl. http://www.dailymail.co.uk/news/article-2300724/Fury-Poland-German-war-drama-tries-spread-blame-Holocaust.html [Zugriff am 01.10.2015].

3 Tomasz Lis: Diesen polnischen Film sollten die Deutschen sehen, in: Die Welt, 15.08.2014, vgl. http://www.welt.de/debatte/kommentare/article131264226/Diesen-polnischen-Film-sollten-die-Deutschen-sehen.html [Zugriff am 01.10.2015].

${ }^{4}$ Florian Peters: Der Warschauer Aufstand in Videoclip-Ästhetik. Der polnische Blockbuster „,Warschau '44" läuft im ZDF-und kaum jemand schaut hin, in: zeitgeschichte-online, http://www. zeitgeschichte-online.de/film/der-warschauer-aufstand-videoclip-aesthetik [Zugriff am 01.10.2015]. 
Infolge des Kalten Krieges kam es in den Jahrzehnten nach dem Zweiten Weltkrieg nur selten zu einer wirklichen wissenschaftlichen Kooperation bei der Bearbeitung von Themen der gemeinsamen Vergangenheit. Erst nach der Öffnung des Eisernen Vorhangs verdichtete sich in Deutschland, in Polen und zwischen den beiden Ländern der Geschichtsdiskurs, der sich nun auf die Jahrhunderte des Zusammenlebens ebenso wie auf die Zeit des nationalsozialistischen Terrors sowie auf Flucht und Vertreibung der Deutschen am Ende und nach dem Zweiten Weltkrieg erstreckte. Wie sich allerdings bald zeigen sollte, basierte dieser Diskurs in den beiden Ländern auf sehr unterschiedlichen Erfahrungs-, Erinnerungs- und Wissenshorizonten.

Die nach 1989 in Wissenschaft, Medien und zum Teil auch in der Politik kontrovers diskutierten Themen bezogen sich auf zahlreiche Aspekte der Landesund Regionalgeschichte im deutsch-polnischen Kontaktbereich und vor allem der deutsch-polnischen Zeitgeschichte. Umstritten war etwa noch immer - wie schon zu Zeiten des Kalten Kriegs - die deutsche bzw. polnische Prägung der früheren Ostgebiete des Deutschen Reichs (die 1945 zu Polens West- und Nordgebieten geworden waren) seit dem Mittelalter oder die Fortdauer ethnozentrischer Sichtweisen in neueren regionalgeschichtlichen Synthesen. Aufgeworfen wurde auch die Frage, ob und inwiefern im deutsch-polnischen Kontext die Akzentuierung der Regional- und Landesgeschichte eine zukunftsweisende Alternative zum traditionellen nationalgeschichtlichen Ansatz darstellen könne. Eine herausgehobene Rolle hatte dabei die seit 1991 von der Kulturgesellschaft „Borussia“ in Bezug auf Ostpreußen (bzw. Ermland und Masuren) ausgehenden Aktivitäten. Aus den Forschungen von Robert Traba ging das Konzept eines „offenen Regionalismus“ hervor, das insbesondere die plurikulturellen und polyethnischen Zusammenhänge dieser Region in den Blick nahm.

Erneut war auch die Rolle Preußens für die Geschichte Polens ein Thema. Diese rückte besonders anlässlich von zwei Jahrestagen - des 300. Jahrestages der preußischen Königskrönung im Jahr 2001 und des 300. Geburtstages König Friedrichs II. des Großen im Jahr 2012 - in den Mittelpunkt. Dabei traten wieder die stark differierenden Preußenbilder in Deutschland und Polen zutage. ${ }^{5}$

Die Hauptaufmerksamkeit der Geschichtsdebatten lag und liegt jedoch auf dem 20. Jahrhundert: Dem Zweiten Weltkrieg, der Okkupation Polens, den Kriegs- und Besatzungsverbrechen, dem Thema Zwangsarbeit sowie auf Flucht und Vertreibung der Deutschen.

Die sich heute, über 25 Jahre nach dem Ende des Kalten Krieges immer noch stellende Frage lautet: Ist es möglich, dass die Geschichte der Deutschen im östlichen Europa von Polen und Deutschen gemeinsam erinnert und zu einem gemeinsamen „lieu de memoiré“ werden kann? Was steht dem noch entgegen? Wie haben

${ }^{5}$ Vgl. Matthias Weber: Adel im östlichen Preußen. In: Historie. Jahrbuch des Zentrums für Historische Forschung der Polnischen Akademie der Wissenschaften 5, 2011/2012, S. 227-246, hier S. $230-232$. 
sich die Geschichtsdiskurse in Deutschland und Polen seit 1989 entwickelt - im Hinblick auf die Erinnerung der Deutschen an die historischen deutschen Ostgebiete und im Hinblick auf die Erinnerung an den Zweiten Weltkrieg und seine Folgen? All das gehört zum Kontext, in dem Kultur und Geschichte der Deutschen im östlichen Europa zu betrachten ist.

$* * *$

Die Überlegungen beziehen sich sowohl auf wissenschaftliche Probleme wie auf Fragen der Erinnerungspolitik und der Geschichtsvermittlung. Seit 2005 ist der Autor gemeinsam mit Partnern insbesondere in Polen, Ungarn, der Slowakei und Rumänien aber auch in Österreich und Tschechien an der inhaltlichen Konzeption und am organisatorischen Aufbau des „Europäischen Netzwerks Erinnerung und Solidarität" beteiligt. Aufgabe dieses Europäischen Netzwerks ist die Bearbeitung und Popularisierung der Geschichte Europas im 20. Jahrhundert, wobei der Akzent auf den totalitären Diktaturen und deren Opfern liegt: In der Arbeit des Netzwerks besitzen der Holocaust, der vom nationalsozialistischen Deutschen Reich begonnene und geführte Vernichtungskrieg in und gegen Polen ebenso wie gegen andere Länder im östlichen Mitteleuropa sowie die Unterdrückung durch die sowjetische Vorherrschaft in den Jahrzehnten nach dem Zweiten Weltkrieg bis 1989 eine herausgehobene Bedeutung. Das „Europäische Netzwerk“ möchte $\mathrm{zu}$ einem Forum des internationalen Vergangenheitsdiskurses werden, dessen Markenzeichen der empathische Blick auf die Erfahrungen der Anderen und die multilaterale Diskussion schwieriger und strittiger historischer Fragen im Geist der Solidarität ist. ${ }^{6}$

Der andere Themenkomplex ist mit dem im Nordwesten Deutschlands gelegenen „Bundesinstitut für Kultur und Geschichte der Deutschen im östlichen Europa“" verbunden, das vom Autor geleitet wird. ${ }^{7}$ Betrachtet wird hier die Vergangenheit von Regionen wie Schlesien (Śląsk), Pommern (Pomorze), Ost- und Westpreußen (Warmia i Mazury, Prusy zachodnie), Böhmen und Mähren sowie die Geschichte der Deutschen im Baltikum, in Siebenbürgen oder in Russland. Es geht hier also um die ehemaligen östlichen Gebiete des Deutschen Reichs und um die früher und teilweise bis heute von Deutschen bewohnten Siedlungsgebiete im östlichen Europa. Auch wenn in der Arbeit dieses Instituts die Geschichte der Deutschen seit dem Mittelalter eine herausgehobene Bedeutung hat, werden sie nicht isoliert, sondern als Mehrheit oder als Minderheit, jedoch stets im Austausch

${ }^{6}$ Europäisches Netzwerk Erinnerung und Solidarität/ European Network Remembrance and Solidarity: „The Idea“, „Goals and Tasks“, vgl. http://www.enrs.eu/en/about-us/ideas.html [Zugriff am 01.10.2015].

${ }^{7}$ Das im Januar 1989 gegründete Bundesinstitut für Kultur und Geschichte der Deutschen im östlichen Europa (BKGE) ist eine wissenschaftliche Einrichtung im Geschäftsbereich der Beauftragten der Bundesregierung für Kultur und Medien; vgl. http://www.bkge.de/ 
und in der Verflechtung mit anderen Ethnien gesehen. Die östlichen Gebiete des Deutschen Reiches wurden 1945 zu den West- und Nordgebieten Polens - Königsberg mit einem Teil Ostpreußens kam an die Sowjetunion und ist heute als „Oblast Kaliningrad“ die kleinste Oblast der russischen Föderation. Die oft mehrfachen nationalen, kulturellen und konfessionellen Identitäten dieser Gebiete werden heute im Rahmen einer Verflechtungsgeschichte betrachtet.

Zur Kultur und Geschichte der Deutschen im östlichen Europa gehört beides: die Jahrhunderte währende gemeinsame Vergangenheit und kulturelle Errungenschaften ebenso wie das von Deutschland und den Deutschen ausgehende Kriegs-, Besatzungs- und Vernichtungsgeschehen des 20. Jahrhunderts.

Nach 1989 ist Deutschland von diesen ,östlichen' Aspekten seiner Vergangenheit eingeholt worden. Zum einen ist die Zeit des Nationalsozialismus und des Zweiten Weltkrieges einschließlich seiner Folgen im öffentlichen Bewusstsein und in den Medien wieder stärker präsent, zum anderen - mit der Öffnung und dem Fall der Grenzen - auch die ältere Kultur und Geschichte der genannten Regionen, in denen seit dem Mittelalter und der frühen Neuzeit die ethnische, nationale, konfessionelle und sprachliche Vielfalt ungleich stärker ausgeprägt war als heute. Karl Schlögel hat bemerkt, dass das Netz der Beziehungen und Kontakte im östlichen Europa, das Deutschland einst selbst zerstört hat, zum Teil wieder neu geknüpft worden sei. ${ }^{8}$ Der damit verbundene Nachholbedarf für das traditionell westorientierte historische und kulturelle Bewusstsein der Deutschen und für die stark nach Westen hin ausgerichtete deutsche Geschichtsschreibung ist immens.

\section{Gegensätzliche Historiographien: Intensivierung des Diskurses nach 1989}

Vor 1989 waren die Geschichtsbilder im Hinblick auf die Geschichte und das Wirken der Deutschen im östlichen Europa im Wesentlichen geordnet. Die Masternarrative in Deutschland und in den östlichen Nachbarstaaten waren deutlich voneinander abgegrenzt und erschienen als geklärt. Etwas vereinfachend zusammengefasst: Auf deutscher Seite waren die Geschichte Schlesiens, Pommerns oder Preußens als Teile der deutschen Landesgeschichte betrachtet worden, wobei ein Akzent auf die Kulturleistungen der Deutschen in Mittelalter und früher Neuzeit gelegt wurde. Unbequeme Themen der Geschichte des 19. und 20. Jahrhunderts, besonders die Zeit des Nationalsozialismus, waren tendenziell vernachlässigt oder ausgeblendet worden. Umgekehrt war es auf Seiten der polnischen Historiographie nicht selten zu einer Überbetonung der Präsenz

${ }^{8}$ Karl Schlögel: Orte und Schichten der Erinnerung. Annäherungen an das östliche Europa, vgl. http://www.eurozine.com/articles/article_2008-12-19-schlogel-de.html [Zugriff am 01.10.2015]. 
polnischer Kultur und Sprache in den genannten Gebieten im Verlauf ihrer Geschichte gekommen. ${ }^{9}$

Die in den neunziger Jahren einsetzenden Diskussionen betrafen nun weniger die Ereignisgeschichte als die ebenso unvermittelt wie unvorbereitet aufeinanderprallenden Narrative. Nach Beendigung des Kalten Krieges und Öffnung der Grenzen wurde in Politik und Medien mit einem Male nicht mehr nur übereinander geschrieben und gesprochen, vielmehr setzte nun erstmals ein direkter, durch keinerlei politische Vorgaben mehr beschränkter Meinungsaustausch über die Vergangenheit ein. Dabei ging es um Reinterpretationen von überkommenen und weit verbreiteten Geschichtsbildern und damit verbunden bald auch um die Gestaltung der Erinnerung im öffentlichen Raum durch Einrichtung neuer Museen und Gedenkstätten.

Dabei wurde deutlich, wie fundamental viele Bilder von der Geschichte des 20. Jahrhunderts im westlichen und im östlichen Europa voneinander abwichen: So zentrale Themen wie die Ermordung der europäischen Juden durch die Nationalsozialisten, die nationalsozialistische Okkupation Polens oder das Ende des Zweiten Weltkrieges hatten in den beiden Ländern unterschiedliches Gewicht: In der Bundesrepublik Deutschland wurde die Debatte darüber, ob das Kriegsende eine katastrophale Niederlage oder eine Befreiung sei, in den neunziger Jahren fortgesetzt. In Polen stand und steht das Jahr 1945 keineswegs nur für das Kriegsende, sondern markiert auch den Übergang von einem diktatorischen Fremdregime zu einem anderen.

Damit wurden Fragen aufgeworfen, die nicht nur die eigene historisch-nationale Identität berührten, sondern die angesichts des verdichteten und europäisierten Diskurses jeweils auch in den Nachbarländern aufmerksam verfolgt wurden und sich sehr bald auf die staatliche Geschichtspolitik auswirkten.

In Deutschland sind nach 1989 gegenläufige Geschichtsdebatten zu beobachten: 1996 löste der Amerikaner Daniel Goldhagen mit seinem Buch „Hitlers willige Vollstrecker"10 eine Debatte über die Verwicklung der ganz gewöhnlichen Deutschen in den Holocaust aus. Ebenfalls 1996 nahm die Ausstellung des Hamburger Instituts für Sozialforschung über die Verbrechen der Wehrmacht Abschied von der Legende von der sauberen Wehrmacht. ${ }^{11}$ Beide Diskussionen führten zu

${ }^{9}$ Vgl. Hackmann, Jörg: Deutschlands Osten-Polens Westen als Problem der Geschichtsschreibung. In: Weber, Matthias (Hrsg.): Deutschlands Osten-Polens Westen. Vergleichende Studien zur geschichtlichen Landeskunde (= Mitteleuropa-Osteuropa. Oldenburger Beiträge zur Kultur und Geschichte Ostmitteleuropas 2). Frankfurt am Main 2001. S. 209-235; Weber, Matthias: Zur historiographischen Bearbeitung der Stellung Schlesiens zwischen dem Heiligen Römischen Reich und den Königreichen Polen und Böhmen. In: Willoweit, Dietmar, Lemberg, Hans (Hrsg.): Reiche und Territorien in Ostmitteleuropa. Historische Beziehungen und politische Herrschaftslegitimation (= Völker, Staaten und Kulturen in Ostmitteleuropa 2). München 2006. S. 13-33.

${ }^{10}$ Goldhagen, Daniel: Hitler's Willing Executioners. New York 1996; deutsch: Hitlers willige Vollstrecker. Berlin 1996.

${ }^{11}$ König, Helmut: Politische und zeithistorische Debatten zur Weltkriegserinnerung im wiedervereinigten Deutschland. In: Troebst, Stefan, Wolf, Johanna (Hrsg.): Erinnern an den Zweiten 
einer noch stärkeren Betonung deutscher Schuld und Täterschaft. Außerdem wurde jetzt auch die bisherige Historiographie über das östliche Europa selbstkritisch reflektiert, ältere zum Teil volks(tums)geschichtlich ausgerichtete Paradigmen wurden hinterfragt und schrittweise beseitigt. Initiale Bedeutung hatte dabei das 1988 erschienene Buch des britischen Historikers Michael Burleigh „Germany Turns Eastwards: A Study of Ostforschung in the Third Reich" ${ }^{\text {"12 }}$, das die Analyse und Dekonstruktion der politisierten sog. „deutschen Ostforschung“ seit der Weimarer Republik anstieß und zahlreiche Folgepublikationen nach sich zog. Burleigh verdeutlichte die zwischen dem Naziregime und den Vertretern der Wissenschaft bestehenden Beziehungen und zeigte, wie die historische Ostmitteleuropaforschung auf die Abwege des biologistischen Rassismus und der nationalsozialistischen Geschichtsmanipulation geriet. Er beschrieb die Zusammenwirkung von Wissenschaft, Politik und SS schon vor dem Krieg bis hin zu den Kontinuitäten der "Ostforschung“ in der zweiten Hälfte des 20. Jahrhunderts.

Mit Beginn des neuen Jahrtausends entstand in Deutschland unvermittelt ein starker Komplementärdiskurs. Schon seit den 1990er Jahren wurde auch das von Deutschen erduldete Kriegsleid unter Akzentuierung des Schicksals der deutschen Vertriebenen ebenso wie der alliierte Bombenkrieg gegen deutsche Städte während und am Ende des Krieges angesprochen. ${ }^{13}$ Nun, über fünf Jahrzehnte nach Kriegsende, trat mit Nachdruck die Erinnerung daran zutage, dass Deutsche im Zweiten Weltkrieg und danach auch Opfer von Grausamkeiten geworden sind. Flucht und Vertreibung und speziell die Frage, „Dürfen Vertriebene Opfer sein?“ wurden explizit aufgeworfen. ${ }^{14}$ Besonders das letztgenannte Thema war zwar bisher nicht tabuisiert worden, es war aber über Jahrzehnte in der Wissenschaft ebenso wie im gesellschaftlichen Bewusstsein eher randständig geblieben. Angestoßen wurde die Diskussion durch den ab 1999 verfolgten Plan des Bundes der Vertriebenen zur Einrichtung eines „Zentrums gegen Vertreibungen“ in Berlin.

Auf der extremen Rechten kam der Begriff „Vertreibungsholocaust“ auf und für die Zerstörung Dresdens die Bezeichnung „Bombenholocaust“, die im Jahr 2005 auf den dritten Rang für das Unwort des Jahres gewählt wurde. ${ }^{15}$

Weltkrieg. Mahnmale und Museen in Mittel- und Osteuropa (= Schriften des Europäischen Netzwerks Erinnerung und Solidarität, Bd. 2). S. 57-64, hier S. 58.

${ }^{12}$ Burleigh, Michael: Germany Turns Eastwards: A Study of Ostforschung in the Third Reich. Cambridge 1988.

${ }^{13}$ Initialisierend wirkte hier das Buch von Jörg Friedrich: Der Brand. Deutschland im Bombenkrieg 1940-1945. München 2002.

${ }^{14}$ Schwartz, Michael: Dürfen Vertriebene Opfer sein? Zeitgeschichtliche Überlegungen zu einem Problem deutscher und europäischer Identität. In: Deutschland Archiv 38 (2005), Heft 3, S. 494-505, hier S. 494.

15 Axel Wermelskirchen: Unwort des Jahres ,,Entlassungsproduktivität“, vgl.: http://www.faz. net/aktuell/feuilleton/unwort-des-jahres-entlassungsproduktivitaet-1306250.html [Zugriff am 01.10.2015]. 
Vielfach meldeten sich Stimmen zu Wort, die eine Viktimisierungstendenz kritisierten: Der Erinnerungstheoretiker Harald Welzer befürchtete einen Rückfall in die Haltung des „leidenden Kollektivs“. Prominente Historiker wir Norbert Frei oder Hans-Ulrich Wehler warnten vor einem erinnerungspolitischen „Gezeitenwechsel“ oder vor einer fatalen „Opferkonkurrenz" zwischen Verfolgten des NS-Staates und Kriegsfolgeopfern. ${ }^{16}$ Es kam auch zu deutsch-polnischen Kontroversen in den Medien ebenso wie zwischen Politikern und Wissenschaftlern sowie in geringerem Umfang zwischen Deutschland und Tschechien. Hinsichtlich Tschechiens fokussierte sich die Diskussion auf den Fortbestand der sog. Beneš-Dekrete des Jahres 1945, auf denen die Zwangsaussiedlung der Deutschen basierte. Ihre Aufhebung wurde insbesondere von den Vertriebenenverbänden ebenso nachhaltig wie vergebens gefordert.

In Polen äußerten kritische Stimmen die Sorge, dass der neue deutsche Geschichtsdiskurs dazu führen könne, dass sich die Deutschen zunehmend selbst als Opfer stilisieren, dass Täter und Opfer vertauscht würden, so dass Polen nicht mehr als das eigentliche Kriegsopfer wahrgenommen, sondern als ,Vertreiberstaat" und damit als Täternation diffamiert werde. Der Historiker Paweł Machcewicz fasste die Problematik so zusammen:

In Polen sprechen wir offen über die dunklen Seiten unserer Geschichte, erinnern an die Leiden, die polnische Hände Bürgern anderer Völker bereitet haben. Dagegen verstärkt sich in Deutschland die Neigung, sich auf das eigene Leid zu konzentrieren, oft in Begleitung ungerechter Anschuldigungen an andere Nationen. In den Schatten gestellt wird die Verantwortung für den Ausbruch des Zweiten Weltkriegs und die verbrecherische Okkupationspolitik als Ursache dessen, was die Deutschen später erlebt haben... ${ }^{17}$

Das Aufeinandertreffen unterschiedlicher historischer Perspektiven bewirkte auch eine erhöhte Sensibilisierung hinsichtlich der Präsentation der Vergangenheit gegenüber der Öffentlichkeit. Soweit die Themen der deutsch-polnischen Konfliktgeschichte des 20. Jahrhunderts betroffen waren, wurde diese im jeweiligen Nachbarland - angesichts zunehmender Diskussion in anderen europäischen Ländern - aufmerksam verfolgt. Es dauerte nicht lange, bis geschichtspolitische Fragen an Bedeutung gewannen und der Vergangenheitsdiskurs eine verstärkte politische, phasenweise sogar tagespolitische Dimension erhielt. ${ }^{18}$

16 Vgl. Schwartz, wie Anm. 14, S. 494.

17 Paweł Machcewicz (2004) Leiter des Büros für öffentliche Bildung des polnischen Instituts für Nationales Gedenken, 11. März 2004, abgedruckt in: Stefan, Troebst (Hrsg.): Vertreibungsdiskurs und europäische Erinnerungskultur. Deutsch-polnische Initiativen zur Institutionalisierung eine Dokumentation (= Veröffentlichung der Deutsch-Polnischen Gesellschaft Bundesverband e.V., Band 11). Osnabrück 2006. S. 118.

18 Pomian, Krzysztof: European identity. Historical fact and political problem (2009), vgl. http://www.eurozine.com/articles/2009-08-24-pomian-en.html [Zugriff am 01.10.2015]; Rydel, Jan: Polityka historyczna w Republice Federalniej Niemiec. Zaszłości, Idee, Praktyka. Uniwersytet Pedagogiczny im. Komisji Edukacji Narodowej - Prace Monograficzne nr 599. Kraków 2011. 
Angeheizt wurde die Geschichtsdebatte durch eine private, in Deutschland aktive Organisation mit der Bezeichnung „Preußische Treuhand“, die versuchte, auf dem Rechtsweg früheres Eigentum von Deutschen in den 1945 an Polen übergegangenen Gebieten zurückzuklagen. Obwohl diese Aktivitäten durch die deutsche Regierung in keiner Weise unterstützt und in Deutschland ganz überwiegend kritisiert wurden, führten sie zu Verunsicherungen in Polen und zu scharfen Gegenreaktionen in der polnischen Presse und Politik. Da die Preußische Treuhand einigen Landsmannschaften der Vertriebenen nahestand, zeigte Polens konservatives Nachrichtenmagazin „Wprost“ in einer skandalösen Fotomontage die Vertriebenenpräsidentin (die sich von der Treuhand distanziert hatte) in einer nationalsozialistischen Uniform auf dem deutschen Bundeskanzler reitend mit der Randbemerkung: „Die Deutschen sind den Polen eine Billion Dollar für den Zweiten Weltkrieg schuldig“. ${ }^{19}$

Im September 2004 verabschiedete der Sejm einstimmig eine Resolution betreffend die „Rechte Polens auf deutsche Kriegsreparationen sowie zu den in Deutschland vorgebrachten unrechtmäßigen Forderungen gegenüber Polen und polnischen Bürgern“ ${ }^{\text {20 }}$ und der damalige Bürgermeister von Warschau verlautbarte, dass 40 Milliarden Dollar als Entschädigung für die Zerstörung Warschaus im Zweiten Weltkrieg noch zu wenig seien. ${ }^{21}$

Polen sah sich im Jahr 2010 sogar mit einer politischen Diskussion über die Mobilmachung und die Kriegsschuldfrage konfrontiert, nachdem die Präsidentin des Bundes der Vertriebenen betont hatte, dass 1939 Polen als erster die Armee mobilisiert hatte und damit andeutete, dass Hitlers Angriff auf Polen lediglich eine Reaktion auf die polnische Mobilisierung gewesen sei. Die Emotionen waren so stark, dass führende deutsche Politiker klarstellen mussten, dass niemand eine Schuld am Zweiten Weltkrieg bei Polen sehen würde. ${ }^{22}$ Solche ebenso irrationalen wie spektakulären Vorgänge waren Symptome der erhöhten Bedeutung von Geschichte und Geschichtspolitik.

In der medialen Geschichtsaufarbeitung in Deutschland wurde nun öfters das Argument genannt, dass man sich nach der vorbildlichen Auseinandersetzung mit der eigenen Schuld gegenüber den Juden und nachdem in der Goldhagen-Debatte

19 „Wprost“" Nr 38, 21.09.2003.

20 Lang, Kai-Olaf: Pragmatic cooperation instead of a strategic partnership. The current status and perspective for German-Polish relations (German Institute for International and Security Affairs. Comments 32, Novemebr 2004), vgl. http://www.swp-berlin.org/fileadmin/contents/products/comments/comments2004_32_lng_ks.pdf [Zugriff am 01.10.2015].

${ }^{21}$ Spiegel-Online 22.09.2004: Umfrage: Polen erwarten von Deutschland Reparationszahlungen, vgl. http://www.spiegel.de/politik/ausland/umfrage-polen-erwarten-von-deutschland-reparationszahlungen-a-319231.html [Zugriff am 01.10.2015].

${ }^{22}$ FAZ.NET, 09.09.2010: Steinbach hat Kriegsschuldfrage nicht bezweifelt, vgl. http://www. faz.net/aktuell/politik/inland/eklat-in-unionsfraktion-steinbach-hat-kriegsschuldfrage-nicht-bezweifelt-11042941.html [Zugriff am 01.10.2015]; Paterson, Tony: Merkel ally quits after claiming Nazis didn 't start war. In: The Independent, 11. September 2010. 
nochmals die deutsche Täterrolle akzentuiert worden sei, nun auch mit gutem Gewissen den eigenen Opfern zuwenden könne und dass die Beschränkung auf die ,ewige Büßerrolle' der Deutschen zu Ende sei. ${ }^{23}$

Die Frage, ob man historische Schuld auch ,vergessen“ dürfe, wurde ganz besonders im Anschluss an die Verleihung des Friedenspreises des Deutschen Buchhandels in der Frankfurter Paulskirche an den Schriftsteller Martin Walser aufgeworfen. Der Preisträger hatte 1998 in seiner Dankesrede über die „Dauerpräsentation unserer Schande" gesprochen und damit den Holocaust gemeint. Nun wurde eingehend darüber debattiert, ob nicht auch unter dieses Thema irgendwann ein „Schlusstrich“ gezogen werden dürfe. ${ }^{24}$

So nahm in Polen vielfach die Sorge zu, die Deutschen wollten sich immer stärker als Opfer und immer weniger als Täter darstellen, während in Deutschland bei Vielen der Eindruck entstand, die Polen wollten den Deutschen das Recht bestreiten, ihrer Opfer zu gedenken. ${ }^{25}$

Im Kalten Krieg war der geschichtliche Rückblick auf „Ostdeutschland“ (damit waren in der deutschsprachigen Historiographie vor 1989 oft nicht die DDR, sondern die ehemaligen Ostprovinzen des Deutschen Reichs gemeint) aber auch auf Themen wie die nationalsozialistische Okkupation in Polen oder Flucht und Vertreibung einfacher gewesen, weil der Widerpart in der Diskussion ja weitgehend gefehlt hatte. Nun jedoch war es nicht leicht, die Geschichtsbilder über diese Regionen um die Perspektiven der polnischen Wissenschaft und Gesellschaft zu erweitern - und die Anerkennung von Schuld gegenüber Polen fiel schwerer als gegenüber Israel. Hinzukamen die Perspektiven der anderen Staaten und Nationen im östlichen Europa. Vereinfacht gesagt: Aus der deutschen Sicht stellte der Terror gegen Polen sozusagen nur eine Facette im Gesamtbild der nationalsozialistischen Verbrechen in Ostmittel- und Osteuropa dar. ${ }^{26}$

In der 1990 der Bundesrepublik Deutschland beigetretenen DDR hatte sich die Situation grundsätzlich anders dargestellt. Die Geschichte der östlichen Provinzen des Deutschen Reichs sowie insbesondere die Themen „Flucht und Vertreibung der Deutschen“" nach dem Zweiten Weltkrieg waren aus politisch-ideologischen Gründen weitgehend ausgeblendet und allenfalls punktuell angesprochen worden. Die Staatsführung der DDR wollte dadurch vermeiden, dass es infolge einer Diskussion dieser Fragen womöglich zu politischen Spannungen mit der

23 Ther, Philipp: Die Last der Geschichte und die Falle der Erinnerung (2006), vgl. http:// www.eurozine.com/articles/article_2006-08-21-ther-de.html [Zugriff am 01.10.2015].

${ }^{24}$ Vgl. Brumlik, Micha / Funke, Hajo / Rensmann, Lars: Umkämpftes Vergessen. Walser-Debatte, Holocaust-Mahnmal und neuere deutsche Geschichtspolitik (= Schriftenreihe Politik und Kultur am Fachbereich Politische Wissenschaft der Freien Universität Berlin). Berlin 2010.

${ }^{25}$ Vgl. Bingen, Dieter: Die deutsch-polnischen Beziehungen nach 1945. In: Aus Politik und Zeitgeschichte 5/6 (2005), S. 9-17, hier S. 16.

26 Ther, Vgl. Ther, wie Anm. 23. 
Volksrepublik Polen als sozialistischem Bruderland kommen könne. Nach 1989 bestand deshalb in den sog. „Neuen Bundesländern“ ein besonderer Informationsbedarf über alle damit zusammenhängenden Fragen.

$$
* * *
$$

Polen definierte sich traditionell vielfach als eine Opfernation, verstand sich sogar als ,Christus unter den Völkern“ aufgrund der geschichtlichen Bedrohungsund Leiderfahrungen: Der Aufteilung des Königreichs Polen durch Preußen, Österreich und Russland am Ende des 18. Jahrhunderts, der Unterdrückung und Niederschlagung des Freiheitskampfes im 19. Jahrhundert, der doppelten Besetzung durch das Deutsche Reich und die Sowjetunion während des Zweiten Weltkriegs, des nationalsozialistischen Terrors, der Niederschlagung des Warschauer Aufstandes und schließlich der unfreien Verhältnisse unter sowjetischer Vorherrschaft nach 1945. Dieser Blick auf die Vergangenheit aus der Perspektive des Opfers wurde nun in Frage gestellt:

Schon seit den 1980er Jahren, besonders nach 1989, öffnete sich in Polen die Diskussion in entgegengesetzter Richtung. Von Historikern angestoßene selbstkritische Debatten über das polnisch-jüdische Verhältnis seit dem 19. Jahrhundert und über das Verhalten von Polen gegenüber Juden während des Zweiten Krieges wurden von den Medien aufgegriffen. Diese Phase der Auseinandersetzung mit den schwierigen Kapiteln der nationalen Geschichte bezog sich auf verschiedene Gruppen (zum Beispiel auch auf die „Aktion Weichsel“ betreffend Zwangsumsiedlung von Ukrainern 1947) und erstreckte sich auch auf das Problem der Vertreibung der Deutschen sowie auf die Problematik der Lager, in denen Deutsche untergebracht wurden.

In den 1990er Jahren fand eine innerpolnische selbstkritische Auseinandersetzung statt, in der die Vertreibungsthematik mit all ihren Implikationen einschließlich der polnischen Verantwortung für das Geschehene erstmals umfassend dokumentiert, diskutiert und aufgearbeitet wurde. ${ }^{27}$ Diese erstreckte sich nicht nur auf die politischen und intellektuellen Eliten, denn wieder sorgten die Medien für eine große Öffentlichkeitsresonanz; möglicherweise befassten sich die Menschen in Polen damals intensiver als in Deutschland mit dem Schicksal der Vertriebenen. ${ }^{28}$

${ }^{27}$ Kraft, Claudia: Die aktuelle Diskussion über Flucht und Vertreibung in der polnischen Historiographie und Öffentlichkeit. In: Zeitgeschichte-online, Thema: Die Erinnerung an Flucht und Vertreibung, Januar 2004, vgl. http://www.zeitgeschichte-online.de/md=Vertreibung-Kraft [Zugriff am 01.10.2015].

${ }^{28}$ Ebd.; als Neuerscheinung zur Thematik ist zu nennen: Müller, Hans-Peter / Zielińska, Maria (Hrsg.): Transgraniczność w perspektiwie socjologicznej. Migracje przymusowe w Europie (= Lubuskie Towarzystwo Naukowe - Seria Monograficzna Tom VIII). Zielona Góra 2012; ferner der Überblick von Włodzimierz Borodziej: Flucht, Vertreibung, Zwangsaussiedlung. In: Lawaty, Andreas / Orłowski, Hubert: Deutsche und Polen: Geschichte, Kultur, Politik. München 2003. S. $88-95$. 
Diese erste große Vertreibungsdebatte in Polen ist in Deutschland allerdings kaum aufgegriffen oder gewürdigt worden - was in Polen wiederum Unverständnis ausgelöst hat. Zur Diskussion über die Vertreibung und Zwangsaussiedlung kam bald ein weiteres problematisches Thema hinzu. 2001 brachte das Buch des amerikanischen Historikers Jan Tomasz Gross ${ }^{29}$ über das Pogrom an jüdischen Einwohnern der Kleinstadt Jedwabne im Nordosten Polens am 10. Juli 1941 zusätzlich den Aspekt der Täterschaft Polens ins Gespräch.

Während Polen also in einigen Bereichen durch eine schmerzhafte Auseinandersetzung mit der Vergangenheit den eigenen Status als Opfernation in Frage stellte und über bisher nie gestellte Fragen eigener Schuld diskutierte, kam es auf deutscher Seite zu neuen Beanspruchungen des Opferstatus - das passte nicht zusammen.

Andrerseits wurden in beiden Ländern auch die Jahrhunderte währenden deutsch-polnischen Kulturbeziehungen in den ehemaligen preußischen Ostprovinzen bzw. den polnischen Westgebieten wiederentdeckt, zumal sich die heutigen Bewohner mit großem Interesse und Empathie auch der deutschen Geschichte ,ihrer' Regionen zuwandten, die Spuren des deutschen Kulturerbes und deutsche Traditionen aufsuchten. Die hieraus entstehenden regionalen Kooperationen haben die deutsch-polnische Zusammenarbeit in praktisch allen geschichts- und kulturwissenschaftlichen Bereichen intensiviert und die Entwicklung von neuen transnationalen Forschungsansätzen und Betrachtungsweisen (unter Stichworten wie ,area studies“, „Transfergeschichte“, „entangled history“, „histoire croisée“, „connected history“, „spatial turn“) begünstigt, welche die Erforschung der Regionalgeschichte ohne nationale Verengungen ermöglichen.

So hat gerade die Befassung mit der Landesgeschichte ausgleichend gewirkt: Im Bereich der Geschichte Schlesiens ist es im Jahr 1999 auf dem 16. Allgemeinen Polnischen Historikertag in Breslau/Wrocław zu einem ersten umfassenden Meinungsaustausch gekommen, als polnische und deutsche Historiker in einer eigenen Sektion über „Umbrüche in der Geschichte Schlesiens diskutierten ${ }^{30}$ und Themen vom Mittelalter bis zum Ende des zweiten Weltkrieges aufgriffen. Das 2004 ins Leben gerufene Periodikum „Śląska Republika Uczonych / Schlesische Gelehrtenrepublik / Slezská Vědecká Obec“ ist das erste wissenschaftliche Periodikum, das die Geschichte Schlesiens explizit aus der deutschen, polnischen und tschechischen Perspektive betrachtet ${ }^{31}$ und die zweibändige deutsch-polnische

${ }^{29}$ Gross, Jan Tomasz: Neighbors. The Destruction of the Jewish Community in Jedwabne, Poland. Princeton 2001.

${ }^{30}$ Ruchniewicz, Krzysztof / Tyszkiewicz, Jakub / Wrzesiński, Wojciech (Hrsg.): XVI Powszechny Zjazd Historyków Polskich, Sekcja 1: Przełomy w dziejach Ślaska. Toruń 2000. S. 111359 .

${ }^{31}$ Fischer, Peter / Kerski, Basil / Röskau-Rydel, Isabel / Ruchniewicz, Krzysztof / Stekel Sabine (Hrsg.): Inter Finitimos - Jahrbuch zur deutsch-polnischen Beziehungsgeschichte, Heft 1, 1992-Heft 11, 2013/14; Hałub, Marek / Mańko-Matysiak, Anna: Ślaska Republika Uczonych / Schlesische Gelehrtenrepublik / Slezská Vědecká Obec. Wrocław, Vol. 1, 2004-Vol. 5, 2012. 
Anthologie „Mein Schlesien, meine Schlesier / Mój Śląsk, moi Ślązacy“32 bietet einen multiperspektivischen Zugang zur Vergangenheit und Gegenwart dieser Region aus der Sicht mehrerer Generationen.

Der 2010 verstorbene Kunsthistoriker Andrzej Tomaszewski (Warschau), der im Jahr 1995 gemeinsam mit seinem Fachkollegen Dethard von Winterfeld (Mainz) den Arbeitskreis deutscher und polnischer Kunsthistoriker ins Leben gerufen hatte, hat den Begriff des ,gemeinsamen Kulturerbes“ ${ }^{\text {33 }}$ in den früher deutschen nun polnischen Regionen geprägt, das es gemeinsam zu entdecken und weiterzuentwickeln gelte. ${ }^{34}$

Als wissenschaftlich besonders innovativ hat sich auch das bereits 1992 gegründete Publikationsforum „Inter Finitimos Jahrbuch zur deutsch-polnischen Beziehungsgeschichte" erwiesen, das über bilaterale Kooperationen informiert. Hinsichtlich der Erforschung von Flucht und Vertreibung der Deutschen bildete die vierbändige deutsch-polnische Quellenedition „Unsere Heimat ist uns ein fremdes Land geworden“/ „Nasza ojczyzna stała sie dla nas obcym państwem...“" den bedeutendsten Fortschritt. ${ }^{35}$ Phasenweise schien es - vor allem im ersten Jahrzehnt des neuen Jahrtausends - dass die deutsch-polnische Vergangenheit mit allen Facetten des Austauschs und der Konflikte zu einem Hauptakzent des Geschichtsdiskurses geworden sei.

\section{Fachdiskurs und gesamtgesellschaftlicher Diskurs}

Debatten über die Geschichte der Deutschen im östlichen Europa, über deutsch-polnische Beziehungsgeschichte und die Konfliktgeschichte des 20. Jahrhunderts waren in Deutschland während der 1990er Jahre zunächst vor allem in Fachkreisen Realität geworden. Im ersten Jahrzehnt des neuen Jahrtausends und im Umfeld des Beitritts Polens und weiterer Staaten zur Europäischen Union (2004 und 2007) wurden historische Themen auch auf dem allgemeinen Buchmarkt und in den Medien stärker präsent. Hier sind exemplarisch die 2002 erschienene

${ }^{32}$ Hałub, Marek / Weber, Matthias (Hrsg.): Mein Schlesien, meine Schlesier. Zugänge und Sichtweisen / Mój Ślask, moi Ślazacy. Eksploracje i obserwacje (= Schlesische Grenzgänger 4 und 6). 2 Bde., Leipzig 2011, 2014.

33 Tomaszewski, Andrzej / von Winterfeld, Dethard (Hrsg.): Wspólne dziedzictwo. Polsko-niemiecka wspótpraca konserwatorska 1970-2000 / Das gemeinsame Kulturerbe. Die deutsch-polnische Zusammenarbeit in der Denkmalpflege 1970-2000. Gniezno 2001.

${ }^{34}$ Omilanowska, Małgorzata: Das Gemeinsame Kulturerbe /Wspólne Dziedzictwo. Warszawa, Vol. 1, 2004 - Vol. IX, 2015.

35 Borodziej, Włodzimierz / Lemberg, Hans (Hrsg.): "Unsere Heimat ist uns ein fremdes Land geworden ..." Die Deutschen östlich von Oder und Neiße 1945-1950. Dokumente aus polnischen Archiven (= Quellen zur Geschichte und Landeskunde Ostmitteleuropas, Bde.1-4). Marburg 20002001; polnische Ausgabe mit dem Titel: "Nasza ojczyzna stała się dla nas obcym państwem ..." Niemcy w Polsce 1945-1950, wybór dokumentów, 4 tomy. Warszawa 2000-2001. 
Novelle von Günter Grass „Im Krebsgang“ zu nennen, die das Thema der Vertreibungen literarisch aufgriff, oder der Film „Die Flucht“ (2007), der die Ereignisse in Ostpreußen im Jahr 1945 verarbeitete sowie zahlreiche Film- und Fernsehdokumentationen über Themen der deutsch-polnischen Geschichte. ${ }^{36}$

In Polen wurde im Verlag DEMART in hoher Auflage der Atlas ,Wysiedlenia, wypędzenia i ucieczki 1939-1959 Atlas ziem Polski“" publiziert, ${ }^{37}$ der unter dem Titel „Zwangsumsiedlung, Flucht und Vertreibung 1939-1959. Atlas zur Geschichte Ostmitteleuropas“ ins Deutsche übersetzt und in großer Auflage über die Bundeszentrale für politische Bildung verteilt wurde. 2010 erschien das bald in die polnische, deutsche und in weitere Sprachen übersetzte Buch „Bloodlands Europe between Hitler and Stalin“ von Timothy Snyder, in dem insbesondere der Kampf und das Leiden der Polen eindrucksvoll geschildert wurde - viele weitere Beispiele könnten genannt werden, ${ }^{38}$ die verdeutlichen, dass viel zur Popularisierung der Geschichte des 20. Jahrhunderts getan wurde.

Mit Hilfe von demoskopischen Forschungen lässt sich zeigen, dass von den differenzierten Geschichtsdebatten in der Wissenschaft, aber auch der intensiven, zum Teil kenntnisreichen Berichterstattung in den Massenmedien keineswegs auf den kollektiven Vergangenheitsdiskurs der Gesamtgesellschaft geschlossen werden kann. Der heutzutage inflationär verwendete Begriff der „kollektiven Erinnerung" ist mit Vorsicht zu gebrauchen, stets unter Berücksichtigung der Frage, auf welches „Kollektiv“ er jeweils bezogen ist: auf Wissenschaft und Fachkreise, auf bestimmte politische oder gesellschaftliche (Interessen-)Gruppen oder tatsächlich auf die gesamte Öffentlichkeit (Allgemeinheit).

Der Blick auf Ergebnisse von zwei Repräsentativumfragen mag dies veranschaulichen: Auf Veranlassung des Hauses der Geschichte der Bundesrepublik Deutschland (Bonn) wurde vom Institut für Demoskopie in Allensbach eine Erhebung durchgeführt, die das Thema „Flucht und Vertreibung aus Sicht der deutschen, polnischen und tschechischen Bevölkerung" untersuchte; die Ergebnisse wurden 2005 in deutscher, polnischer und tschechischer Sprache publiziert. ${ }^{39}$ 2011 veröffentlichte die Stiftung für Deutsch-Polnische Zusammenarbeit (Warschau) ebenfalls die Ergebnisse einer in Deutschland und in Polen durchgeführten repräsentativen Allensbach-Umfrage über den Stand der deutsch-polnischen Be-

${ }^{36}$ Grass, Günter: Im Krebsgang. Göttingen 2002.

37 Wysiedlenia, wypędzenia i ucieczki 1939-1959. Atlas ziem Polski. Warszawa 2008; deutsche Ausgabe: Zwangsumsiedlung, Flucht und Vertreibung 1939-1959. Atlas zur Geschichte Ostmitteleuropas (Bundeszentrale für politische Bildung, Schriftenreihe 1015). Bonn 2010.

38 Snyder, Timothy: Bloodlands. Europe between Hitler and Stalin. New York 2010; deutsche Ausgabe: Bloodlands. Europa zwischen Hitler und Stalin (2011); polnische Ausgabe: Skrwawione ziemie: Europa między Hitlerem i Stalinem (2011).

39 Petersen, Thomas: Flucht und Vertreibung aus Sicht der deutschen, polnischen und tschechischen Bevölkerung, hrsg. von Haus der Geschichte der Bundesrepublik Deutschland. Bonn 2005. [= Umfrage 2005] 
ziehungen unter dem Motto: „Ein großer Schritt in Richtung Normalität“، ${ }^{40}$ Die Ergebnisse lassen sich wie folgt zusammenfassen:

Das Thema der geschichtlichen deutsch-polnischen Beziehungen bildet für die deutsche Gesamtgesellschaft offenbar keinen wirklich herausgehobenen „lieu de mémoiré“. Die Kenntnisse über das Nachbarland sind (unverändert) gering und lückenhaft, in der Schule spielen die Geschichte Polens und des östlichen Europas kaum eine Rolle. Die Umfrage von 2005 ergab beispielsweise, dass auf einer Europakarte ohne Ortsnamen nur etwa 18 Prozent der Deutschen die Lage Schlesiens annähernd richtig eintragen konnte, wobei ,annähernd richtig“" großzügig ausgelegt wurde. Nur etwa zehn Prozent der Deutschen besitzen elementare historische, geographische oder zahlenmäßige Kenntnisse über die Thematik „Flucht und Vertreibung der Deutschen“" am Ende des Zweiten Weltkrieges. ${ }^{41}$ So ist es nicht überraschend, dass Kenntnisse über Themen der polnischen Vergangenheit wie die Besatzung Polens in der Zeit des Nationalsozialismus, den Warschauer Aufstand oder über die Tatsache, dass Polen im 20. Jahrhundert unter zwei Totalitarismen zu leiden hatte, in der deutschen Gesellschaft kaum vorhanden sind besonders nicht unter der jüngeren Bevölkerung. Nur 22 Prozent aller Deutschen können heutzutage noch eine besondere, aus dem Zweiten Weltkrieg resultierende Verantwortung gegenüber Polen erkennen - je jünger die Befragten sind, desto höher wird dieser Anteil. ${ }^{42}$

Für die polnischen Durchschnittskenntnisse bezogen auf die Gesamtgesellschaft ergeben sich in Teilen ähnliche Befunde: Die Allensbach-Studie von 2005 ergibt, dass auch die Kenntnisse vieler Polen über die Vergangenheit des heutigen West- und Nordpolen und über die früher hier wohnenden Deutschen eher gering sind. In einer Liste mit den Namen der Städte Warszawa (Warschau), Łódź (Lodsch), Kraków (Krakau), Wrocław (Breslau), Szczecin (Stettin) und Gdańsk (Danzig) konnte nur die Hälfte der polnischen Bevölkerung Gdańsk, Wrocław und Szczecin dem ehemals deutschen Siedlungsgebiet zuordnen, wobei übrigens die Kenntnisse der Befragten in den West- und Nordgebieten selbst nicht wesentlich besser waren als im übrigen Polen. ${ }^{43}$

Die Umfrage von 2011 bestätigt, dass die Erinnerung an und die Kenntnisse über den Zweiten Weltkrieg sowie das Bewusstsein der von den Deutschen während des Zweiten Weltkrieges in Polen und seinen Menschen begangenen Kriegsverbrechen in Polen generationenübergreifend unverändert viel stärker ausgeprägt

${ }^{40}$ Fundacja Współpracy Polsko-Niemieckiej / Stiftung für Deutsch-Polnische Zusammenarbeit: Ein großer Schritt in Richtung Normalität: Der Stand der deutsch-polnischen Beziehungen. Ergebnisse repräsentativer Bevölkerungsumfragen in Deutschland und Polen. Institut für Demoskopie Allensbach 2011 [= Umfrage 2011].

${ }^{41}$ Umfrage 2005, wie Anm. 16, S. 29-31.

42 Umfrage 2011, wie Anm. 17, S. 47; vgl. Umfrage 2005, wie Anm. 16, S. 76.

43 Umfrage 2005, wie Anm. 16, S. 76. 
sind als in Deutschland. ${ }^{44}$ Deshalb bewertet die polnische Bevölkerung das Verhältnis zu Deutschland heute durchaus anders als die Deutschen das Verhältnis zu Polen. 54 Prozent aller Polen sind der Meinung, Deutschland habe gegenüber Polen auch heute noch eine besondere Verpflichtung, die sich aus den Verbrechen des Zweiten Weltkrieges ableitet. ${ }^{45}$ Zwischen den Spezialisten beider Länder (also den deutschen Poleninteressenten und den polnischen Deutschlandinteressenten) gibt es hingegen keine Unterschiede - jeweils knapp über die Hälfte ist der Ansicht, dass eine solche Verpflichtung besteht.

2015 wurde vom Allensbacher Institut für Demoskopie eine weitere Umfrage $^{46}$ durchgeführt, nun speziell zum Thema „Flucht, Vertreibung, Versöhnung““ Ergebnis ist, dass „Flucht und Vertreibung“ infolge des Zweiten Weltkriegs die Deutschen heute mehr beschäftigen als vor zehn Jahren, was vor allem auf die derzeitige weltweite Aktualität der Migrationsproblematik zurückgeführt wird. Allerdings sehen 52 Prozent der Polen die bilateralen Beziehungen durch das Thema „Flucht und Vertreibung“ als belastet an, aber nur 16 Prozent der Deutschen. Hier besteht also eine Asymmetrie fort, obwohl die bilateralen deutsch-polnischen Beziehungen von einer Mehrheit von über 70 Prozent auf beiden Seiten als gut oder sehr gut eingestuft werden.

Insgesamt lässt sich aus den Umfragen folgendes Fazit ziehen: Das Interesse der Deutschen an Polen und umgekehrt ist groß - aber es wird im Blick auf die Gesamtgesellschaft eben nicht primär aus der Vergangenheit oder durch die historischen Beziehungen der beiden Staaten geprägt. Entscheidend für das kollektive Bild der Gesellschaften von einander, für Sympathie oder Antipathie, sind vielmehr die jeweils aktuellen, tagespolitischen Entwicklungen der deutsch-polnischen Beziehungen in Europa, also die Nachrichten über wirtschaftliche Zusammenarbeit, Entwicklung des Arbeitsmarkts, Erfolge im Sport, in der Wissenschaft, der Kultur oder anderen gesellschaftlichen Bereichen. Geschichtsbilder sind auch von Konjunkturen abhängig.

\section{Instrumentalisierungen der Geschichte}

Aus der Komplexität der Vergangenheit, unterschiedlichen historischen Erfahrungen, dem relativ großen wechselseitigen Interesse gepaart mit lückenhaften

${ }^{44}$ Vgl. auch Ruchniewicz, Krzysztof: Die Erinnerung an den Zweiten Weltkrieg in Polen (2007), http://www.eurozine.com/articles/article_2007-09-05-ruchniewicz-de.html\#footNote2 [Zugriff am 01.10.2015].

45 Vgl. Umfrage 2011, wie Anm. 17, S. 48.

46 Institut für Demoskopie Allensbach: Flucht, Vertreibung, Versöhnung. Zusammenfassung der wichtigsten Ergebnisse einer repräsentativen Bevölkerungsumfrage in Deutschland, Polen und Tschechien, Juni 2015, http://www.sfvv.de/sites/default/files/downloads/zusammenfassung_allensbach_studie_sfvv.pdf [Zugriff am 01.10.2015]. 
historischen Kenntnissen und asymmetrischen Erinnerungen erwächst die Gefahr, dass die Geschichte in gesellschaftlichen und politischen Auseinandersetzungen instrumentalisiert und unter Umständen auch missbraucht wird. Beides ist nach 1989 wiederholt geschehen:

In den ersten Jahren nach 1989, besonders nach Unterzeichnung des Vertrages zwischen der Bundesrepublik Deutschland und der Republik Polen über gute Nachbarschaft und freundschaftliche Zusammenarbeit im Jahr 1991, entstand zunächst eine enthusiastische Phase der Versöhnung zwischen Deutschen und Polen.

1995 hielt der polnische Außenminister Władysław Bartoszewski eine wichtige, in Deutschland sehr positiv aufgenommene Rede im Deutschen Bundestag, in der die schwierigen Fragen der deutsch-polnischen Geschichte deutlich angesprochen und am Schluss eine Betrachtung aus der europäischen Perspektive gefordert wurde. ${ }^{47}$

Das Bedürfnis der Versöhnung manifestierte sich damals in ganz unterschiedlichen Inszenierungen, Gesten, Begegnungsveranstaltungen und Publikationen. Sie standen oft für eine allgemeine, europäisierende Lesart der Vergangenheit, die nicht selten unangenehmere Seiten der Beziehungsgeschichte ausblendete und die deutsch-polnischen Gemeinsamkeiten und den Kulturaustausch über die Jahrhunderte beschwor. Dabei ging es nicht selten vor allem um eine moralische Entlastungsfunktion. Der Historiker und Politologe Klaus Bachmann hat dies 1994 als „Versöhnungskitsch“48 zwischen Deutschen und Polen bezeichnet und kritisiert, dass der Enthusiasmus und der Mangel an ernsthaften bilateralen Gesprächen eine Quelle künftiger Probleme werden könne ${ }^{49}$. An diese euphorische Phase schloss sich tatsächlich eine Phase an, in der unterschiedliche Positionen aufeinander prallten.

Dabei stand der erwähnte Vorschlag des Bundes der Vertriebenen zur Errichtung eines „Zentrums gegen Vertreibungen“ in Berlin, im Fokus. Kritiker sahen in dem Projekt einen Versuch, die deutsche Schuld an den nationalsozialistischen Verbrechen in Polen zu relativieren und zu minimalisieren ${ }^{50}$ sowie der Installierung eines neuen Geschichtsdiskurses, durch den Opfer und Täter vertauscht wür-

47 Die Rede von Władysław Bartoszewski im Deutschen Bundestag am 28. April 1995 im Internet: http://www.bundestag.de/kulturundgeschichte/geschichte/gastredner/bartoszewski/rede bartoszewski.html\#barto [Zugriff am 01.10.2015].

${ }^{48}$ Bachmann, Klaus: Die Versöhnung muss von Polen ausgehen. In: Tageszeitung 5. August 1994, Hahn, Hans, Henning / Hein-Kircher, Heidi / Kochanowska-Nieborak, Anna (Hrsg.): Erinnerungskultur und Versöhnungskitsch, Marburg 2008 (= Tagungen zur Ostmitteleuropa-Forschung, Bd. 26). Marburg 2008.

49 Bachmann, Klaus, 1994, nach Jarząbek, Wanda: Shadows of Memory in Polish-German Relations (1989-2005). In: Kopp, Kristin / Niżyńska, Joanna (Hrsg.): Germany, Poland, and postmemorial relations. In search of a livable past (= Europe in Transition: The NYU European Studies Series). New York 2012. S. 25-42, hier S. 37.

50 Jarząbek, wie Anm. 49, S. 34. 
den. In einer 2003 unter anderem von polnischen, deutschen und tschechischen Historikern unterzeichneten Stellungnahme wurde die Kritik zusammengefasst:

Dabei sehen wir vor allem zwei Gefahren in historischer und politischer Dimension. Historisch betrachtet droht eine Entkontextualisierung der Vergangenheit, die Negation des ursächlichen Zusammenhangs von NS-Volkstums- und Vernichtungspolitik auf der einen und Flucht und Vertreibung der Deutschen auf der anderen Seite. Die politische Gefahr besteht insbesondere in der Ethnisierung von gesellschaftlichen Konflikten, also der Umdeutung von politischen und sozialen Kontroversen in ethnische - und damit der Zementierung eines völkischen Verständnisses von Vergangenheit, Gegenwart und Zukunft. ${ }^{51}$

Der im Jahr 2005 geschlossene Koalitionsvertrag der Regierungsparteien in Deutschland enthielt den Auftrag, in Berlin ein „Sichtbares Zeichen“"zu errichten, „um an das Unrecht von Vertreibungen zu erinnern und Vertreibung für immer zu ächten“. Die Fortsetzung der Diskussion betraf die Konzeption dieses „Sichtbaren Zeichens", das schließlich von der Bundesregierung in Form der staatlichen Stiftung „Flucht, Vertreibung, Versöhnung“ umgesetzt wurde. Die Kritik aus Polen auch an diesem Projekt hielt zunächst an, obwohl „Versöhnung“ als Stiftungsziel im Gesetzestext verankert war. ${ }^{52}$ Es bestand die Sorge, dass die Zeit des Nationalsozialismus und die Verbrechen der Deutschen in Polen nicht angemessen dargestellt, dass das Leiden der Deutschen überhöht und Geschichte „umgeschrieben“ werde. ${ }^{53}$ Schließlich konnte aber 2012 eine inhaltlich multiperspektivische Konzeption verabschiedet werden, die im In- und Ausland akzeptiert wurde. ${ }^{54}$

Die historische Frage, ob das 20. Jahrhundert als ,Jahrhundert der Vertreibungen“ oder als ,Jahrhundert der totalitären Diktaturen“ zu bezeichnen sei, und die methodische Frage, wie man „Vertreibung europäisch erinnern“"55 kann, wurden in den Medien auf beiden Seiten immer wieder mit überwunden geglaubten wech-

${ }^{51}$ For a critical and enlightened debate about the past, 10. August 2003, vgl. http://www. vertreibungszentrum.de/ [Zugriff am 01.10.2015].

52 Stiftung Flucht, Vertreibung, Versöhnung, vgl. http://www.sfvv.de [Zugriff am 01.10.2015]; Dokumentation der Gazeta Wyborcza, vgl. http://info.wyborcza.pl/temat/wyborcza/fundacja $+u-$ cieczka+wyp\%C4\%99dzenie+pojednanie [Zugriff am 01.10.2015]. Im Stiftungsgesetz wird betont: Zweck der Stiftung ist es, ,im Geiste der Versöhnung die Erinnerung und das Gedenken an Flucht und Vertreibung im 20. Jahrhundert im historischen Kontext des Zweiten Weltkrieges und der nationalsozialistischen Expansions- und Vernichtungspolitik und ihrer Folgen wachzuhalten“.

${ }^{53}$ Kittel, Manfred: Czy Niemcy rzeczywiście chca napisać inna historię. Zadania i cele berlińskiej fundacji “Ucieczka, Wypędzenie, Pojednanie”. In: Müller, Hans-Peter / Zielińska, Maria (Hrsg.): Transgraniczność w perspektywie socjologicznej. Migracje przymusowe w Europie (= Lubuskie Towarzystwo Naukowe - Seria Monograficzna Tom VIII). Zielona Góra 2012. S. 335-344.

${ }^{54}$ Konzeption für die Arbeit der Stiftung Flucht, Vertreibung, Versöhnung und Leitlinien für die geplante Dauerausstellung, vgl. http://www.sfvv.de/sites/default/files/downloads/conceptual_ framework_sfvv_2012.pdf [Zugriff am 01.10.2015].

55 Bingen, Dieter / Borodziej, Wlodzimierz / Troebst, Stefan (Hrsg.): Vertreibungen europäisch erinnern? Historische Erfahrungen - Vergangenheitspolitik - Zukunftskonzeptionen (= Veröffentlichungen des Deutschen Polen-Instituts 18). Wiesbaden 2003; Naimark, Norman: Fires of hatred. Ethnic Cleansing in Twentieth-Century Europe. Harvard 2001; Mann, Michael: The Dark Side of Democracy. Explaining Ethnic Cleansing. Cambridge 2004. 
selseitigen Stereotypen angereichert und die unterschiedliche Beurteilung der Vergangenheit wurde auch auf andere politische Felder übertragen:

In Polen wurde die vermeintliche neue Bedrohung durch den veränderten deutschen Geschichtsdiskurs vielfach als politisches Argument genutzt. Zum Beispiel im Wahlkampf von 2005, als behauptet wurde, der Großvater des damaligen Kandidaten Donald Tusk habe im Zweiten Weltkrieg in der deutschen Wehrmacht gedient. Oder 2006, als der damalige polnische Verteidigungsminister Sikorski die Pläne zum Bau einer russisch-deutschen Ostsee-Gaspipeline in die Tradition des Ribbentrop-Molotow-Paktes rückte ${ }^{56} .2011$ wurde im Wahlkampf das Bild vom militaristischen deutschen „Drang nach Osten“ bemüht, um die gefühlte Bedrohung durch die Vertriebenenverbände zu verdeutlichen, die Befürchtung, jene beabsichtigten Deutschland zu einer imperialen Politik der Rückgewinnung der ehemals deutschen Gebiete im Westen und Norden Polens zu veranlassen. Die deutsche Massenpresse wiederum griff das Bild der ewig unzufriedenen „Opfernation“ auf, die nicht aufhören will, Entschädigung für Kriegsverluste zu fordern. ${ }^{57}$ - man hatte eigentlich angenommen, dass derartige Argumente selbst Geschichte geworden seien.

\section{Abgleichen der Geschichtsbilder}

Welche Folgerungen sind aus den skizzierten Diskursen in Deutschland und Polen zu ziehen? Trotz des tiefgreifenden Wandels seit dem Jahr 1989 ist auch im heutigen Europa die „Nation“ der primäre Bezugspunkt für die Erinnerungsgemeinschaften geblieben und nichts deutet darauf hin, dass sich dies in absehbarer Zeit ändern wird. Der Ausgleich der Asymmetrien in den Erinnerungskulturen der europäischen Nationen wird deshalb auch künftig eine kultur- und gesellschaftspolitisch wichtige Aufgabe bleiben.

Heute ist im politischen Raum und besonders in der Kulturförderung sehr viel von der ,europäischen Erinnerungskultur', einem ,gemeinsamen europäischen Geschichtsverständnis‘ und einer ,europäischen Identität‘ die Rede, die es zu entwickeln gelte. Mit derartigen Forderungen sollte meiner Ansicht nach eher zurückhaltend umgegangen werden, zumal wenn es um Ostmitteleuropa, um die Geschichte der Deutschen in diesem Raum oder um die deutsch-polnischen Be-

${ }^{56}$ Miodek, Marcin: „Das ist ein neuer Ribbentrop-Molotow-Pakt! “. Eine historische Analogie in der polnischen Energiedebatte. In: Osteuropa 59. Jg., Heft 7-8 (2009), S. 295-305.

57 Beispiele in den Beiträgen von Thomas Urban: Vertreibung als Thema in Polen und von Thomas Speckmann: Renaissance des Themas in den Medien “. In: Stiftung Haus der Geschichte der Bundesrepublik Deutschland (Hrsg.): Flucht, Vertreibung Integration-Begleitbuch zur Ausstellung. Bonn 2005, S. 157-165 und 175-179; Kaczynski Warns of Germany's 'Imperial' Ambitions (2011), vgl. http://www.spiegel.de/international/europe/polish-opposition-leader-kaczynski-warns-of-germany-s-imperial-ambitions-a-790034.htm [Zugriff am 01.10.2015]. 
ziehungen allgemein geht: Der Versuch einer Normierung des Blicks auf die Vergangenheit ist durch die Geschichte selbst bereits endgültig diskreditiert worden. Demokratisch verfasste und pluralistisch geprägte Gesellschaften benötigen keine vereinheitlichten Geschichtsbilder, die - realistisch betrachtet - aufgrund der extrem gegensätzlichen Erfahrungen und Perspektiven sowie der Vielschichtigkeit des Gegenstandes ohnehin nicht erreichbar sind.

Wirklich notwendig ist eine verbesserte Verständigung der Staaten und Gesellschaften über ihre unterschiedlichen Geschichtsbilder. Dies gilt insbesondere dann, wenn sich der Stand der wissenschaftlichen Auseinandersetzung weit von gesellschaftlichen Geschichtsbildern entfernt hat, wie es zum Teil gerade im Bereich der historischen Ostmitteleuropaforschung der Fall ist. Realistisch und wünschenswert erscheint es, um mit Dan Diner zu sprechen, dass sich allmählich die „nationalen Gedächtnisse [...] untereinander abgleichen“58 und dass es dadurch wechselseitig zu einer Ergänzung bisheriger oft unvollständiger Geschichtsbilder und zum Abbau von tradierten Geschichtsmythen kommt. ${ }^{59}$

Wenn sich Vertreter unterschiedlicher nationaler oder anderweitiger Diskursgemeinschaften verständigen können, Geschichtsbetrachtung von der Ereignisebene auf die Ebene der Narrative (auf die „Geschichte zweiten Grades“) zu heben, dann entsteht unter ihnen ein Grundkonsens, der die Kommunikation über die unterschiedlichen Zugänge und Sichtweisen ermöglicht. Wenn so allmählich eine von Empathie geleitete Erinnerungspolyphonie an das 20. Jahrhundert entsteht, wenn etwa Täter-Opfer-Diskurse sachlich geführt werden, ohne die jeweiligen Positionen politischen Richtungen oder Interessengruppen zuzuordnen, werden einseitige Geschichtsdeutungen als Zweckargument auch für den politischen Streit an Bedeutung verlieren. Ziel sollte es sein, die in der Wissenschaft längst erkannte Polyphonie und Multiperspektivität der Erinnerung an die Vergangenheit stärker als bisher in den Gesellschaften Deutschlands, Polens und anderer Staaten Europas zu vermitteln.

\section{Literatur}

Bingen, Dieter / Borodziej, Wlodzimierz / Troebst, Stefan (Hrsg.): Vertreibungen europäisch erinnern? Historische Erfahrungen - Vergangenheitspolitik - Zukunftskonzeptionen (= Veröffentlichungen des Deutschen Polen-Instituts 18). Wiesbaden 2003.

58 Diner, Dan: Gedächtnis und Restitution. In: Knigge, Volkhard / Frei, Norbert (Hrsg.): Verbrechen Erinnern. Die Auseinandersetzung mit Holocaust und Völkermord. München 2002. S. 299305, hier S. 304.

59 Weber, Matthias / Olschowsky, Burkhard / Petranský, Ivan A. / Pók, Attila / Przewoźnik, Andrzej (Hrsg.): Erinnerungsorte in Ostmitteleuropa - Erfahrungen der Vergangenheit und Perspektiven (= Schriften des Bundesinstituts für Kultur und Geschichte der Deutschen im östlichen Europa, Bd. 42; Schriften des Europäischen Netzwerks Erinnerung und Solidarität, Bd. 1). München 2011. 
Borodziej, Włodzimierz: Flucht, Vertreibung, Zwangsaussiedlung. In: Lawaty, Andreas / Orłowski, Hubert: Deutsche und Polen: Geschichte, Kultur, Politik. München 2003. S. 88-95.

Borodziej, Włodzimierz / Lemberg, Hans (Hrsg.): "Unsere Heimat ist uns ein fremdes Land geworden ..." Die Deutschen östlich von Oder und Neiße 1945-1950. Dokumente aus polnischen Archiven (= Quellen zur Geschichte und Landeskunde Ostmitteleuropas, Bde.1-4). Marburg 2000-2001.

Brumlik, Micha / Funke, Hajo / Rensmann Lars: Umkämpftes Vergessen. Walser-Debatte, Holocaust-Mahnmal und neuere deutsche Geschichtspolitik (= Schriftenreihe Politik und Kultur am Fachbereich Politische Wissenschaft der Freien Universität Berlin). Berlin 2010.

Bundeszentrale für politische Bildung: Zwangsumsiedlung, Flucht und Vertreibung 1939-1959. Atlas zur Geschichte Ostmitteleuropas (= Schriftenreihe 1015). Bonn 2010.

Burleigh, Michael: Germany Turns Eastwards: A Study of Ostforschung in the Third Reich. Cambridge 1988.

Bingen, Dieter: Die deutsch-polnischen Beziehungen nach 1945. In: Aus Politik und Zeitgeschichte 5/6 (2005), S. 9-17.

Diner, Dan: Gedächtnis und Restitution. In: Knigge, Volkhard, Frei, Norbert (Hrsg.): Verbrechen Erinnern. Die Auseinandersetzung mit Holocaust und Völkermord. München 2002. S. 299-305.

Fischer, Peter / Kerski, Basil / Röskau-Rydel, Isabel / Ruchniewicz, Krzysztof / Stekel, Sabine: Inter Finitimos - Jahrbuch zur deutsch-polnischen Beziehungsgeschichte, Heft 1, 1992-Heft 11, 2013/14.

Friedrich, Jörg: Der Brand. Deutschland im Bombenkrieg 1940-1945. München 2002.

Fundacja Współpracy Polsko-Niemieckiej / Stiftung für Deutsch-Polnische Zusammenarbeit: Ein großer Schritt in Richtung Normalität: Der Stand der deutsch-polnischen Beziehungen. Ergebnisse repräsentativer Bevölkerungsumfragen in Deutschland und Polen. Allensbach 2011 [= Umfrage 2011].

Goldhagen, Daniel: Hitler's Willing Executioners. New York 1996.

Grass, Günter: Im Krebsgang. Göttingen 2002.

Gross, Jan Tomasz: Neighbors. The Destruction of the Jewish Community in Jedwabne, Poland. Princeton 2001.

Hackmann, Jörg: Deutschlands Osten-Polens Westen als Problem der Geschichtsschreibung. In: Weber, Matthias (Hrsg.): Deutschlands Osten-Polens Westen. Vergleichende Studien zur geschichtlichen Landeskunde. (= Mitteleuropa-Osteuropa. Oldenburger Beiträge zur Kultur und Geschichte Ostmitteleuropas 2). Frankfurt am Main 2001. S. 209-235.

Hahn, Hans Henning / Hein-Kircher, Heidi / Kochanowska-Nieborak, Anna (Hrsg.): Erinnerungskultur und Versöhnungskitsch (= Tagungen zur Ostmitteleuropa-Forschung, Bd. 26). Marburg 2008.

Hałub, Marek / Mańko-Matysiak, Anna: Śląska Republika Uczonych / Schlesische Gelehrtenrepublik / Slezská Vědecká Obec, Vol. 1. Wrocław 2004.

Hałub, Marek / Mańko-Matysiak, Anna: Śląska Republika Uczonych / Schlesische Gelehrtenrepublik / Slezská Védecká Obec, Vol. 5. Wrocław 2012.

Hałub, Marek / Weber, Matthias (Hrsg.): Mein Schlesien, meine Schlesier. Zugänge und Sichtweisen / Mój Ślask, moi Ślazacy. Eksploracje i obserwacje (= Schlesische Grenzgänger 4). Leipzig 2011.

Hałub, Marek / Weber, Matthias (Hrsg.): Mein Schlesien, meine Schlesier. Zugänge und Sichtweisen / Mój Ślask, moi Ślazacy. Eksploracje i obserwacje (= Schlesische Grenzgänger 6). Leipzig 2014.

Jarząbek, Wanda: Shadows of Memory in Polish-German Relations (1989-2005). In: Kopp, Kristin / Niżyńska, Joanna (Hrsg.): Germany, Poland and postmemorial relations. In search of a livable past (= Europe in Transition: The NYU European Studies Series). New York. 2012. S. 25-42.

Kittel, Manfred: Czy Niemcy rzeczywiście chca napisać inna historię. Zadania i cele berlińskiej fundacji "Ucieczka, Wypędzenie, Pojednanie”. In: Müller, Hans-Peter / Zielińska, Maria (Hrsg.): Transgraniczność w perspektywie socjologicznej. Migracje przymusowe w Europie 
(= Lubuskie Towarzystwo Naukowe - Seria Monograficzna Tom VIII). Zielona Góra 2012. S. 335-344.

König, Helmut: Politische und zeithistorische Debatten zur Weltkriegserinnerung im wiedervereinigten Deutschland. In: Troebst, Stefan / Wolf, Johanna (Hrsg.): Erinnern an den Zweiten Weltkrieg. Mahnmale und Museen in Mittel- und Osteuropa (= Schriften des Europäischen Netzwerks Erinnerung und Solidarität, Bd. 2). S. 57-64.

Mann, Michael: The Dark Side of Democracy. Explaining Ethnic Cleansing. Cambridge 2004.

Miodek, Marcin: „,Das ist ein neuer Ribbentrop-Molotow-Pakt! “. Eine historische Analogie in der polnischen Energiedebatte. In: Osteuropa 59. Jg., Heft 7-8, 2009. S. 295-305.

Müller, Hans-Peter / Zielińska, Maria (Hrsg.): Transgraniczność w perspektiwie socjologicznej. Migracje przymusowe $w$ Europie (= Lubuskie Towarzystwo Naukowe - Seria Monograficzna Tom VIII). Zielona Góra 2012.

Naimark, Norman: Fires of hatred. Ethnic Cleansing in Twentieth-Century Europe. Harvard 2001.

Omilanowska, Małgorzata: Das Gemeinsame Kulturerbe / Wspólne Dziedzictwo, Vol. 1. Warszawa 2004.

Omilanowska, Małgorzata: Das Gemeinsame Kulturerbe / Wspólne Dziedzictwo, Vol. IX. Warszawa 2015.

Paterson, Tony: Merkel ally quits after claiming Nazis didn't start war. In: The Independent, 11. September 2010.

Petersen, Thomas: Flucht und Vertreibung aus Sicht der deutschen, polnischen und tschechischen Bevölkerung. Bonn 2005. [=Umfrage 2005]

Ruchniewicz, Krzysztof / Tyszkiewicz, Jakub / Wrzesiński, Wojciech (Hrsg.): XVI Powszechny Zjazd Historyków Polskich, Sekcja 1: Przełomy w dziejach Śląka. Toruń 2000. S. 111-359.

Rydel, Jan: Polityka Historyczna w Republice Federalniej Niemiec. Zaszłości, Idee, Praktyka (= Uniwersytet Pedagogiczny im. Komisji Edukacji Narodowej - Prace Monograficzne nr 599). Kraków 2011.

Schwartz, Michael: Dürfen Vertriebene Opfer sein? Zeitgeschichtliche Überlegungen zu einem Problem deutscher und europäischer Identität. In: Deutschland Archiv 38 (2005), Heft 3, S. 494-505.

Snyder, Timothy: Bloodlands. Europe between Hitler and Stalin. New York 2010.

Snyder, Timothy: Bloodlands. Europa zwischen Hitler und Stalin. München 2011.

Snyder, Timothy: Skrwawione ziemie: Europa między Hitlerem i Stalinem. Warszawa 2011.

Thomas Speckmann: Renaissance des Themas in den Medien. In: Stiftung Haus der Geschichte der Bundesrepublik Deutschland (Hrsg.): Flucht, Vertreibung, Integration - Begleitbuch zur Ausstellung. Bonn 2005. S. 175-179.

Tomaszewski, Andrzej / von Winterfeld, Dethard (Hrsg.): Wspólne dziedzictwo. Polsko-niemiecka wspólpraca konserwatorska 1970-2000 / Das gemeinsame Kulturerbe. Die deutsch-polnische Zusammenarbeit in der Denkmalpflege 1970-2000. Gniezno 2001.

Urban, Thomas: Vertreibung als Thema in Polen. In: Stiftung Haus der Geschichte der Bundesrepublik Deutschland (Hrsg.): Flucht, Vertreibung, Integration - Begleitbuch zur Ausstellung. Bonn 2005. S. 157-165.

Weber, Matthias: Adel im östlichen Preußen. In: Historie. Jahrbuch des Zentrums für Historische Forschung der Polnischen Akademie der Wissenschaften 5, 2011/2012, S. 227-246.

Weber, Matthias: Germans in Eastern Europe as a Polish-German Lieu de mémoire. On the Asymmetry of Memories. In: Pakier, Małgorzata / Wawrzyniak, Joanna (Hrsg.): European Memories - The Eastern Perspective (= Contemporary European History). New York 2015. S. 264-282.

Weber, Matthias: Zur historiographischen Bearbeitung der Stellung Schlesiens zwischen dem Heiligen Römischen Reich und den Königreichen Polen und Böhmen. In: Willoweit, Dietmar / Lemberg, Hans (Hrsg.): Reiche und Territorien in Ostmitteleuropa. Historische Beziehungen und politische 
Herrschaftslegitimation (= Völker, Staaten und Kulturen in Ostmitteleuropa 2). München 2006. S. 13-33.

Weber, Matthias / Olschowsky, Burkhard / Petranský, Ivan A. / Pók, Attila / Przewoźnik, Andrzej (Hrsg.): Erinnerungsorte in Ostmitteleuropa - Erfahrungen der Vergangenheit und Perspektiven (= Schriften des Bundesinstituts für Kultur und Geschichte der Deutschen im östlichen Europa, Bd. 42; Schriften des Europäischen Netzwerks Erinnerung und Solidarität, Bd. 1). München 2011.

\section{Internetquellen}

"Nasze matki, nasi ojcowie" - skandal czy serial poruszajacy, vgl. http://wyborcza. pl/1,75968,14141962,_Nasze_matki_nasi_ojcowie__skandal_czy_serial_poruszajacy.html\#ixzz2c7sHd300 [Zugriff am 01.10.2015].

Die Rede von Władysław Bartoszewski im Deutschen Bundestag am 28. April 1995 im Internet: http://www.bundestag.de/kulturundgeschichte/geschichte/gastredner/bartoszewski/rede_bartoszewski.html\#barto [Zugriff am 01.10.2015].

Dokumentation der Gazeta Wyborcza, vgl. http://info.wyborcza.pl/temat/wyborcza/fundacja $+u-$ cieczka+wyp\%C4\%99dzenie+pojednanie [Zugriff am 01.10.2015].

Europäisches Netzwerk Erinnerung und Solidarität/ European Network Remembrance and Solidarity: „The Idea“, „Goals and Tasks“, vgl. http://www.enrs.eu/en/about-us/ideas.html [Zugriff am 01.10.2015].

FAZ.NET, 09.09.2010: Steinbach hat Kriegsschuldfrage nicht bezweifelt, vgl. http://www.faz.net/ aktuell/politik/inland/eklat-in-unionsfraktion-steinbach-hat-kriegsschuldfrage-nicht-bezweifelt-11042941.html [Zugriff am 01.10.2015].

Florian Peters: Der Warschauer Aufstand in Videoclip-Ästhetik. Der polnische Blockbuster ,Warschau '44" läuft im ZDF - und kaum jemand schaut hin, in: zeitgeschichte-online, http:// www.zeitgeschichte-online.de/film/der-warschauer-aufstand-videoclip-aesthetik [Zugriff am 01.10.2015].

For a critical and enlightened debate about the past, 10. August 2003, vgl. http://www.vertreibungszentrum.de/

http://umuv.zdf.de/Unsere-M\%C3\%BCtter-unsere-V\%C3\%A4ter/Unsere-M\%C3\%BCtter-unsere-V\%C3\%A4ter-26223848.html [Zugriff am 01.10.2015].

http://www.bkge.de/

http://www.eurozine.com/articles/2009-08-24-pomian-en.html [Zugriff am 01.10.2015].

Institut für Demoskopie Allensbach: Flucht, Vertreibung, Versöhnung. Zusammenfassung der wichtigsten Ergebnisse einer repräsentativen Bevölkerungsumfrage in Deutschland, Polen und Tschechien, Juni 2015, http://www.sfvv.de/sites/default/files/downloads/zusammenfassung_allensbach_studie_sfvv.pdf [Zugriff am 01.10.2015].

Kaczynski Warns of Germany's 'Imperial' Ambitions (2011), vgl. http://www.spiegel.de/international/europe/polish-opposition-leader-kaczynski-warns-of-germany-s-imperial-ambitions-a-790034.htm [Zugriff am 01.10.2015].

Konzeption für die Arbeit der Stiftung Flucht, Vertreibung, Versöhnung und Leitlinien für die geplante Dauerausstellung, vgl. http://www.sfvv.de/sites/default/files/downloads/conceptual_ framework_sfvv_2012.pdf [Zugriff am 01.10.2015].

Kraft, Claudia: Die aktuelle Diskussion über Flucht und Vertreibung in der polnischen Historiographie und Öffentlichkeit, in: Zeitgeschichte-online, Thema: Die Erinnerung an Flucht und Vertreibung, Januar 2004, vgl. http://www.zeitgeschichte-online.de/md=Vertreibung-Kraft [Zugriff am 01.10.2015]. 
Lang, Kai-Olaf: Pragmatic cooperation instead of a strategic partnership. The current status and perspective for German-Polish relations (German Institute for International and Security Affairs. Comments 32, November 2004), vgl. http://www.swp-berlin.org/fileadmin/contents/products/comments/comments2004_32_lng_ks.pdf [Zugriff am 01.10.2015].

Lis, Tomasz: Diesen polnischen Film sollten die Deutschen sehen, in: Die Welt, 15.08.2014, vgl. http://www.welt.de/debatte/kommentare/article131264226/Diesen-polnischen-Film-sollten-die-Deutschen-sehen.html [Zugriff am 01.10.2015].

Pomian, Krzysztof: European identity. Historical fact and political problem (2009), vgl. http://www. eurozine.com/articles/2009-08-24-pomian-en.html [Zugriff am 01.10.2015].

Robson, Steve: Fury in Poland over German war drama which 'tries to spread blame for Holocaust', vgl. http://www.dailymail.co.uk/news/article-2300724/Fury-Poland-German-war-drama-triesspread-blame-Holocaust.html [Zugriff am 01.10.2015].

Ruchniewicz, Krzysztof: Die Erinnerung an den Zweiten Weltkrieg in Polen (2007), vgl. http:// www.eurozine.com/articles/article_2007-09-05-ruchniewicz-de.html\#footNote2 [Zugriff am 01.10.2015].

Schlögel, Karl: Orte und Schichten der Erinnerung. Annäherungen an das östliche Europa, vgl. http:// www.eurozine.com/articles/article_2008-12-19-schlogel-de.html [Zugriff am 01.10.2015].

Spiegel-Online 22.09.2004: Umfrage: Polen erwarten von Deutschland Reparationszahlungen, vgl. http://www.spiegel.de/politik/ausland/umfrage-polen-erwarten-von-deutschland-reparationszahlungen-a-319231.html [Zugriff am 01.10.2015].

Stiftung Flucht, Vertreibung, Versöhnung, vgl. http://www.sfvv.de [Zugriff am 01.10.2015].

Ther, Philipp: Die Last der Geschichte und die Falle der Erinnerung (2006), vgl. http://www.eurozine.com/articles/article_2006-08-21-ther-de.html [Zugriff am 01.10.2015].

Wermelskirchen, Axel: Unwort des Jahres „Entlassungsproduktivität“, vgl. http://www.faz.net/ aktuell/feuilleton/unwort-des-jahres-entlassungsproduktivitaet-1306250.html, [Zugriff am 01.10.2015].

\section{Abstracts}

Der Beitrag befasst sich mit zwei Bereichen: der deutschen und polnischen Auseinandersetzung mit der gemeinsamen Vergangenheit ebenso wie mit der deutsch-polnischen Konfliktgeschichte des 20. Jahrhunderts. Er argumentiert für eine wechselseitige Abgleichung, Ergänzung und Vervollständigung der Geschichtsbilder, ohne zu versuchen, Vereinheitlichungen oder gar Standardisierungen vorzunehmen; dabei werden wissenschaftliche Fragen ebenso wie praktische Probleme der Gestaltung von kollektiver Erinnerung angesprochen.

Schlüsselwörter: Geschichte 20. Jahrhundert, Zweiter Weltkrieg, Erinnerung, Deutsch-polnische Beziehungen, Geschichte als Argument 


\title{
Culture and history of the Germans in Eastern Europe in German-Polish debate. On the asymmetry of memories
}

This paper deals with both past and current German and Polish historical debate on the common past and experiences, and especially the history of conflict in the twentieth century. It argues for a reconciliation and the mutual completion of historical analysis and views of history. It argues likewise for contrasting approaches to become attuned to and supplement one another without seeking to standardize the consideration of historical issues. Perspectives of scholarly as well as of practical questions of memory are reflected.

Keywords: history of the 20th century, World War II, remembrance, German-Polish relations, history as argument

\author{
Matthias Weber \\ Direktor \\ Bundesinstitut für Kultur und Geschichte \\ der Deutschen im östlichen Europa \\ Johann-Justus-Weg 147 \\ D-26 127 Oldenburg \\ Deutschland \\ E-Mail: matthias.weber@bkge.uni-oldenburg.de
}

